\title{
Circulating tumor cell count as a biomarker of a specific gastric cancer subgroup characterized by bone metastasis and/or disseminated intravascular coagulation - an early indicator of chemotherapeutic response
}

\author{
MASAHIRO INOUE, KAZUNORI OTSUKA and HIROYUKI SHIBATA \\ Department of Clinical Oncology, Graduate School of Medicine, Akita University, Akita 010-8543, Japan
}

Received February 14, 2015; Accepted October 28, 2015

DOI: $10.3892 / \mathrm{ol} .2015 .4056$

\begin{abstract}
The incidence of gastric cancer coupled with multiple bone metastases, and/or disseminated intravascular coagulation (DIC), is characterized by the clinical presentation of rapid progression and a poor prognosis, and differs from typical gastric cancers. Circulating tumor cells (CTCs) are negligible in typical advanced gastric cancers, however, a considerable number of CTCs in the bloodstream may be detected in the subgroup demonstrating multiple bone metastases and/or DIC. The present study analyzed two cases, with the first case regarding a 51-year-old male who exhibited a CTC count of 275 cells $/ 7.5 \mathrm{ml}$ following an initial, ineffective, chemotherapy cycle. The patient underwent a second chemotherapy course that was effective, and the cell count was observed to reduced to 2 cells $/ 7.5 \mathrm{ml}$. A decreased CTC count was first confirmed on day 16 following treatment. During the chemoresistant phase, the CTC count was observed to increase again. The second case presented by the current study describes a 59-year-old female who exhibited a CTC count of 235 cells/7.5 $\mathrm{ml}$ prior to chemotherapy. This subsequently decreased to 7 cells $/ 7.5 \mathrm{ml}$ following an effective course of chemotherapy. Notably, the CTC count increased alongside disease progression in this case. Within the rare subgroup of gastric cancer patients with multiple bone metastases and/or DIC, CTC count may serve as an early biomarker allowing the evaluation of therapeutic efficacy. However, due to the
\end{abstract}

Correspondence to: Professor Hiroyuki Shibata, Department of Clinical Oncology, Graduate School of Medicine, Akita University, Hondo 1-1-1, Akita 010-8543, Japan

E-mail: hiroyuki@med.akita-u.ac.jp

Abbreviations: CTC, circulating tumor cell; DIC, disseminated intravascular coagulation; PTX, paclitaxel; CEA, carcinoembryonic antigen; CA19-9, carbohydrate antigen 19-9; DTX, docetaxel; CT, computed tomography

Key words: circulating tumor cells, bone metastasis, disseminated intravenous coagulation, gastric cancer, biomarker aggressive nature of this type of cancer, imaging analysis is not recommended as it may typically take several months to complete.

\section{Introduction}

Advanced gastric cancer may present with various clinical manifestations. Visceral metastases, including those of the liver and lung, arise with high incidence in numerous cases of gastric cancer; however, bone metastasis occurs at a lower frequency $(<10 \%)(1,2)$. Advanced gastric cancer with the presence of multiple bone metastases and/or disseminated intravascular coagulation (DIC) is also rare. The frequency of comorbidities with DIC in gastric cancer patients who have bone metastasis is as high as $82-86 \%(3,4)$. The frequency of bone metastasis in gastric cancer patients with DIC is also high at $87 \%$ (5). The existence of this gastric cancer subgroup bearing bone metastasis with susceptibility to DIC has been previously recognized $(3,5)$. The clinical features of this specific subgroup reportedly differ from those presenting with typical gastric cancer, with the DIC subgroup much less likely to develop visceral metastases to the liver and lungs. However, the cancer is much more aggressive and patients have a poorer prognosis. There are several reports of this subgroup in the current literature $(3,5)$. The median survival time ranges from 8 to 22 weeks $(3,5)$, and there are currently no established specific chemotherapies. It is believed that the subgroup of gastric cancer with bone metastasis and DIC may have a different underlying biological mechanism, and that chemotherapy regimens against this may have different requirements. Methotrexate plus 5-fluorouracil is effective in controlling the gastric cancer with metastasis and/or DIC subgroup $(4,6)$; however, there is limited information regarding the responses of recently approved agents, including taxanes, cisplatin, S1 and capecitabine (7-10).

The aggressive nature and progression of this subgroup often leaves patients without the chance to undergo a second treatment option. Furthermore, it is difficult to measure the response to specific therapeutics due to the discrepancy of tumor marker levels and the time-consuming nature of imaging analyses. Among the associated biomarkers, circulating tumor cell (CTC) count is a direct indicator of tumor growth. 

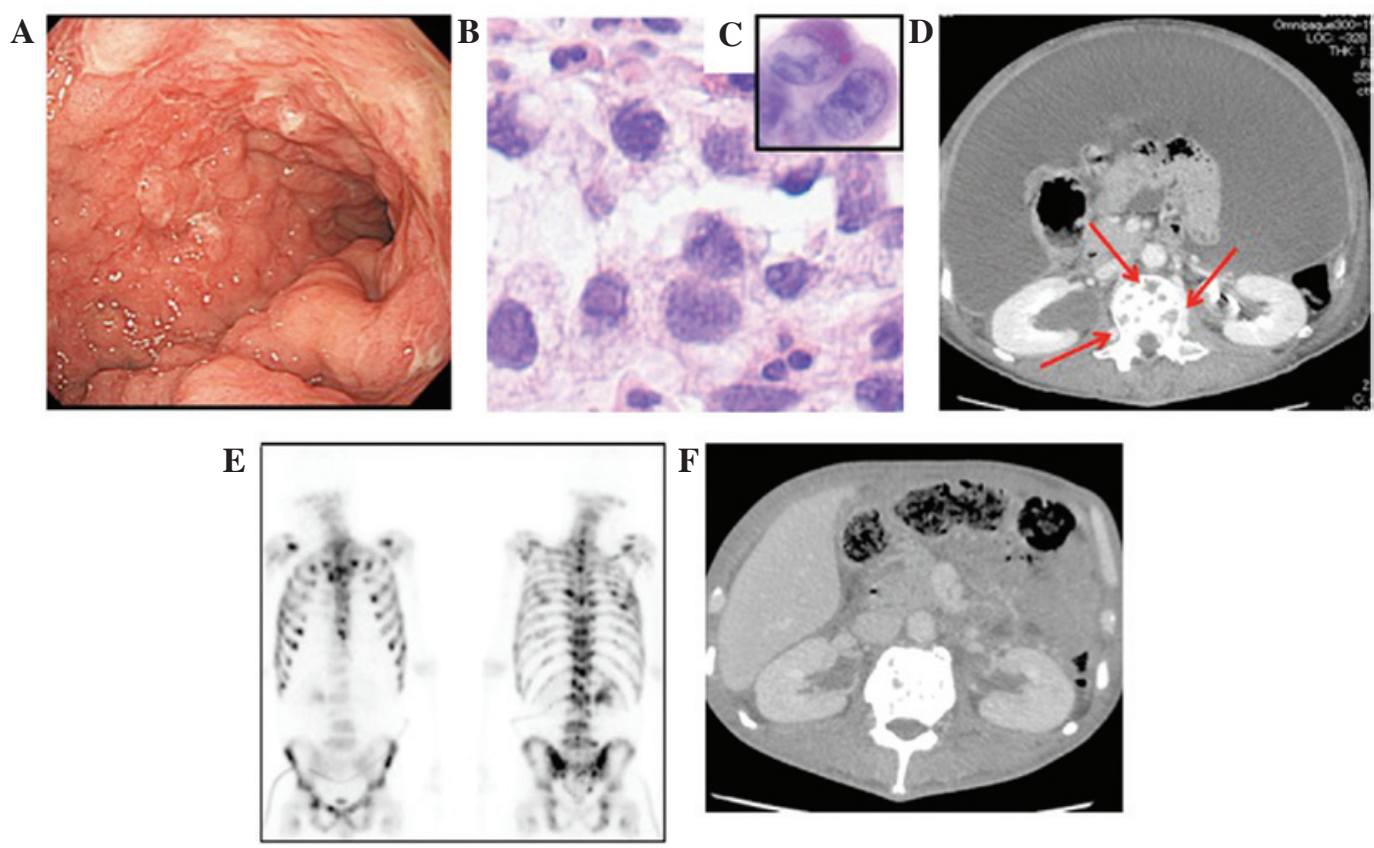

Figure 1. Clinicopathological features of case 1. (A) Endoscopic view. (B) A pathological view of a gastric tumor. (C) Cytology of ascites. (D) CT on day 2 following the initial treatment. Red arrow indicates bone metastases (osteolytic region). (E) Bone scintigram. (F) CT on day 68. CT, computed tomography.

A

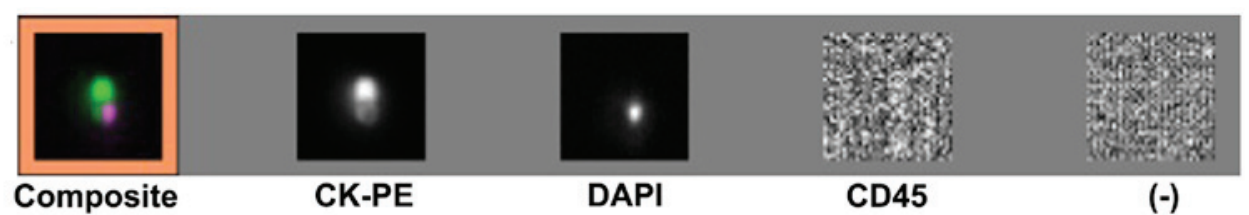

B

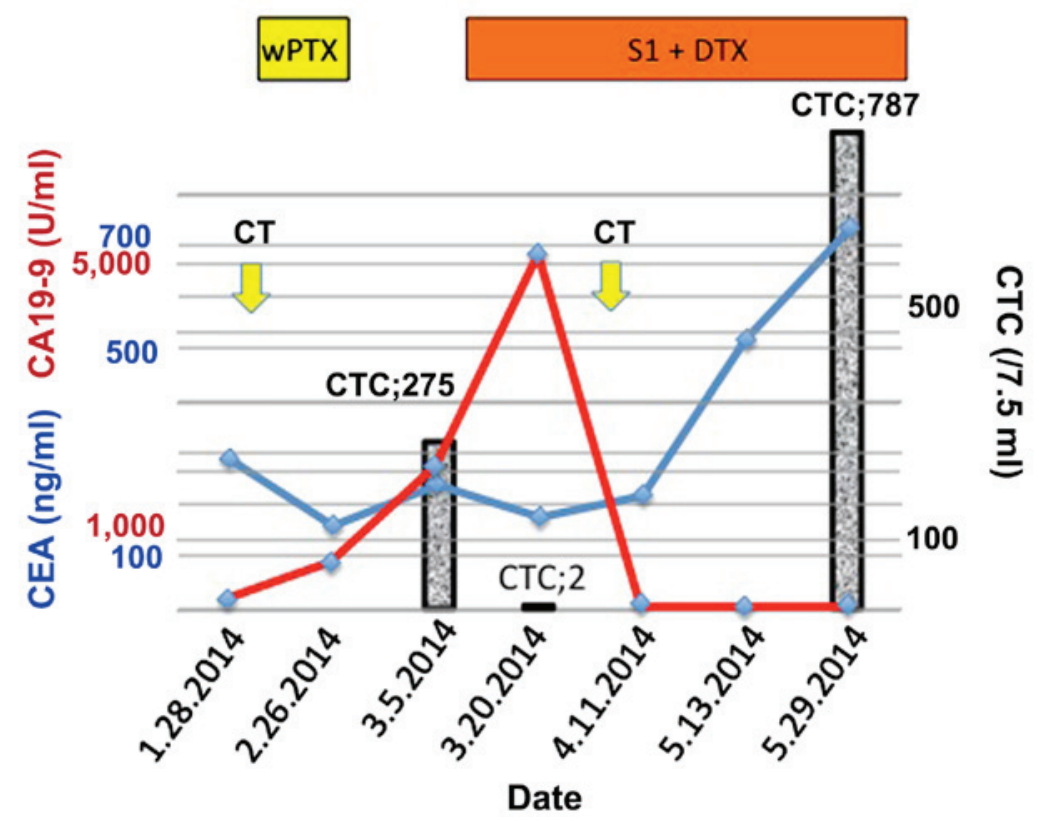

Figure 2. Clinical course of case 1. (A) Appearance of the captured CTC. The composite image of CTC stained with CK-PE and DAPI is presented representatively. Cluster of differentiation 45 (leukocyte common antigen) is negative in the CTC. (-) indicates the background. (B) Clinical course of case 1. Captured CTC count is indicated by the bar graph. CTC, circulating tumor cell; CK-PE, pan-cytokeratin antibody; DAPI, 4',6-diamidino-2-phenylindole; PTX, paclitaxel; DTX, docetaxel; CEA, carcinoembryonic antigen; CA19-9, carbohydrate antigen 19-9.

Despite gastric cancer not generally being associated with high CTC counts (median, 2 cells/7.5 ml) (11), this particular subgroup of gastric cancer with multiple bone metastases and/or DIC is believed to exhibit high CTC counts. However, there are currently no reports confirming this. The present study describes the CTC count in two patients that fall within 
A

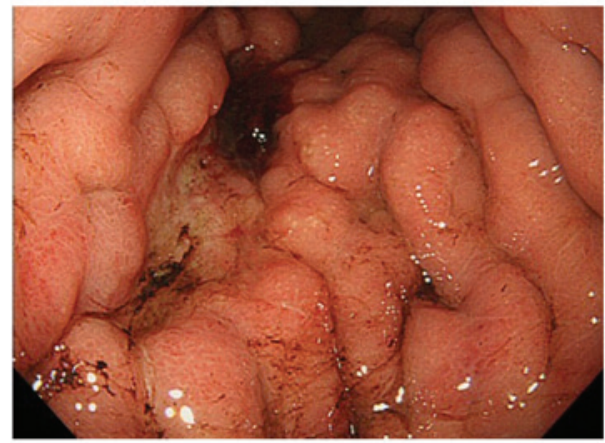

B

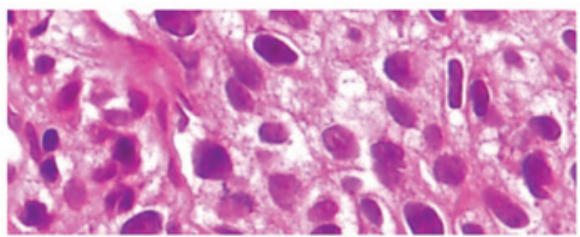

C

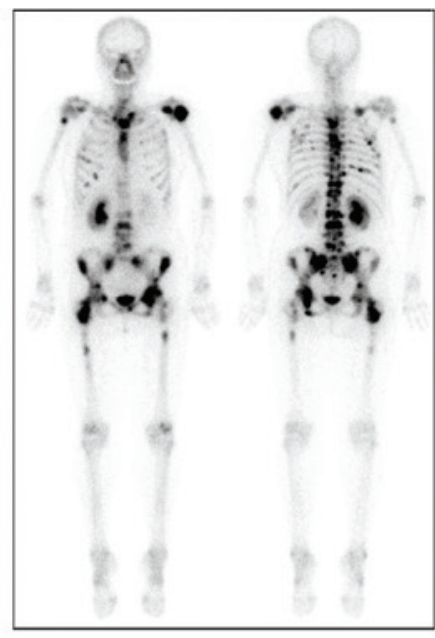

E

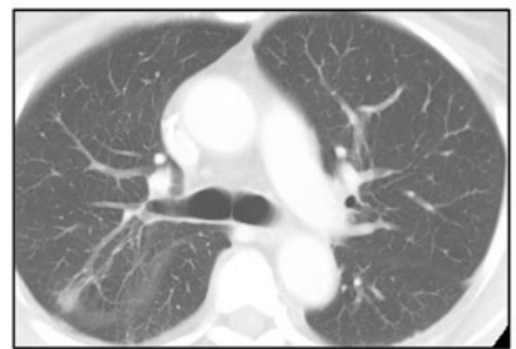

G

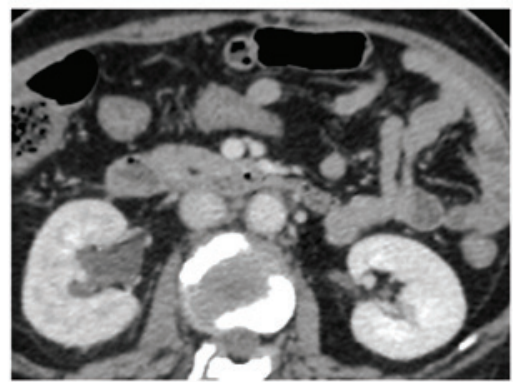

Figure 3. Clinicopathological features of case 2. (A) Endoscopic view. (B) Pathological view of gastric tumor. (C) Bone scintigram. (D) Chest CT on day 17. (E) Chest CT on day 96. (F) Abdominal CT on day 17. Red dots indicate lymph node metastases. (G) CT on day 96. CT, computed tomography.

the advanced gastric cancer with metastasis and/or DIC subgroup, and investigate its possible function as a clinical biomarker. The present observational study was approved by the Ethics Committee of the School of Medicine of Akita University (Akita, Japan). Written informed consent and an agreement to publish were obtained from each patient.

\section{Case report}

Case 1. In January 2014, a 51-year-old male patient presented to the Department of Clinical Oncology, Akita University Hospital (Akita, Japan) and was diagnosed with scirrhous-type gastric cancer, with bone metastases to the thoracic and lumbar vertebrates and multiple costae, in addition to malignant ascites (Fig. 1). Poorly-differentiated adenocarcinoma and signet ring cell carcinoma were detected in the stomach, and ascites was noted (Fig. 1B and C). A left nephrostomy was performed due to the occurrence of hydronephrosis and enabled the administration of chemotherapy. Recombinant thrombomodulin $\alpha(380 \mathrm{U} / \mathrm{kg})$ was administered for 4 days to improve DIC, and concomitant chemotherapy with paclitaxel (PTX; 45-80 mg/m², weekly) was administered for 3 weeks. The platelet count immediately increased, but the ascites did not improve. Following one cycle of PTX, the carcinoembryonic antigen (CEA) level decreased from 288.7 to $160.2 \mathrm{ng} / \mathrm{ml}$ (normal range, $4.9 \mathrm{ng} / \mathrm{ml}$ ), but the carbohydrate antigen 19-9 (CA19-9) level increased from 158.3 to $690.5 \mathrm{U} / \mathrm{ml}$ (normal range, $37.0 \mathrm{U} / \mathrm{ml}$ ). Additionally, the CTC count was calculated as 275 cells $/ 7.5 \mathrm{ml}$ during the same time period (Fig. 2). A previously described method was used to isolate the CTCs $(12,13)$. In brief, CTCs were isolated from $20 \mathrm{ml}$ of peripheral venous blood drawn using a CellSearch ${ }^{\circledR}$ Circulating Tumor Cell kit and a CellTracks ${ }^{\circledR}$ AutoPrep ${ }^{\circledR}$ system (Janssen Diagnostics; Johnson \& Johnson, New Brunswick, NJ, USA). This procedure was outsourced to Special Reference Laboratories, Inc. (Tokyo, Japan). The drugs were changed and a regimen of $\mathrm{S} 1\left(40 \mathrm{mg} / \mathrm{m}^{2}\right.$, twice daily, for 14 days) plus docetaxel (DTX; $33 \mathrm{mg} / \mathrm{m}^{2}$ ) were administered. Following this, on the 17th day, the CTC count decreased to 2 cells/7.5 ml, but the CA19-9 level remained elevated at 5,150.8 U/ml (Fig. 2B). On day 22, the CA19-9 level decreased to $1,808.8 \mathrm{U} / \mathrm{ml}$, and finally, it reached $58.0 \mathrm{U} / \mathrm{ml}$ 


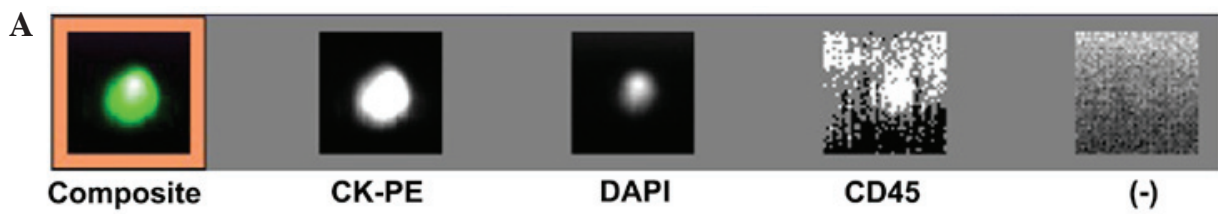

B

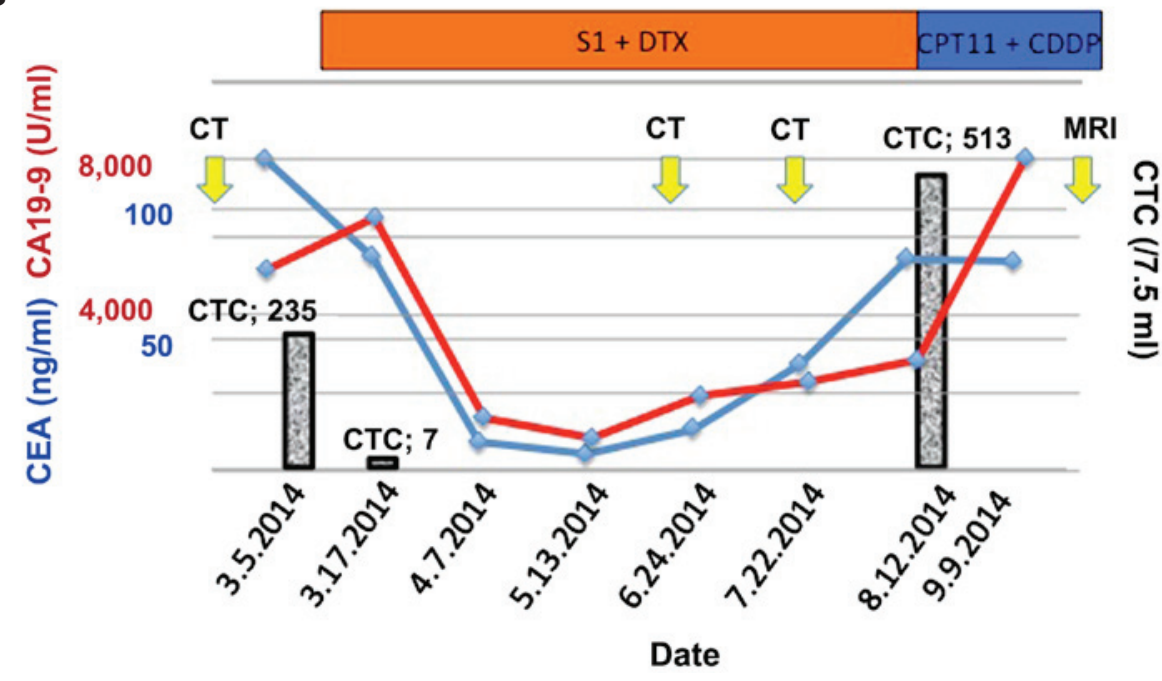

Figure 4. Clinical course of case 2. (A) Appearance of the captured CTC. The composite image of CTC stained with CK-PE and DAPI is presented representatively. Cluster of differentiation 45 (leukocyte common antigen) is negative in the CTC. (-) indicates the background. (B) Clinical course of case 2. CTC is indicated by the bar graph. DTX, docetaxel; CK-PE, pan-cytokeratin antibody; CTC, circulating tumor cell; DAPI, 4',6-diamidino-2-phenylindole; MRI, magnetic resonance imaging; CT, computed tomography; CD45, cluster of differentiation 45; CDDP, cisplatin; CPT11, irinotecan.

on day 35. Computed tomography (CT) imaging confirmed the concomitant disappearance of ascites (Fig. 1F). This state was consistent for 1.5 months, during which the patient was treated as an outpatient. Two months after the initiation of the second chemotherapy course, the CA19-9 level remained suppressed, although the CEA level increased to $733.7 \mathrm{ng} / \mathrm{ml}$, and the CTC count increased to 787 cells $/ 7.5 \mathrm{ml}$. Subsequently, the chemotherapy regimen was changed to cisplatin $\left(23 \mathrm{mg} / \mathrm{m}^{2}\right)$ plus irinotecan $\left(47 \mathrm{mg} / \mathrm{m}^{2}\right)$, but failed. The patient survived for a total of 160 days, but finally succumbed to renal failure due to cancerous peritonitis.

Case 2. In February 2014, a 59-year-old female presented to the Department of Clinical Oncology, Akita University Hospital, and was diagnosed with scirrhous-type gastric cancer, with bone metastases to the thoracic and lumbar vertebrates, the left shoulder joint, the bilateral iliac bones and the right hip joint, in addition to multiple lymph node metastases (Fig. 3). A poorly-differentiated adenocarcinoma was detected at an enlarged gastric fold using a gastrointestinal fibroscope biopsy (Fig. 3B). During the first examination, the patient suffered from dyspnea caused by pulmonary lymphangitis carcinomatosa (Fig. 3D). Two days prior to initiating the first course of chemotherapy, the CTC count was noted at 235 cells $/ 7.5 \mathrm{ml}$. The CEA and CA19-9 levels were also elevated, at $120.0 \mathrm{ng} / \mathrm{ml}$ and $5,201.5 \mathrm{U} / \mathrm{ml}$, respectively. Chemotherapy with $\mathrm{S} 1$ (40 $\mathrm{mg} / \mathrm{m}^{2}$, twice daily, for 14 days) plus DTX $\left(40 \mathrm{mg} / \mathrm{m}^{2}\right)$ was then initiated. In order to efficiently evaluate the therapeutic effect, CTC counts and tumor marker measurements were conducted on day 11 following the initiation of chemotherapy. Discrepant results for CEA $(82.5 \mathrm{ng} / \mathrm{ml})$ and CA19-9 (6,543.2 U/ml) levels were obtained. Conversely, the CTC count had decreased to 7 cells/7.5 ml (Fig. 4). The S1 plus DTX treatment was continued, and subsequently, the CA19-9 level decreased to $1,393.1 \mathrm{U} / \mathrm{ml}$ on day 31 . By day 68 , the CA19-9 and CEA levels had decreased to $835.2 \mathrm{U} / \mathrm{ml}$ and $5.9 \mathrm{ng} / \mathrm{ml}$, respectively. On day 95, CT confirmed that the abdominal lymph node swelling had resolved and the lymphangitis had improved (Fig. 3E and G). However, following day 110 , levels of the two markers began to increase, and on day 159, the levels of CA19-9 and CEA reached 2,858.4 U/ml and $81.7 \mathrm{ng} / \mathrm{ml}$, respectively. Despite the Response Evaluation Criteria in Solid Tumors (14) by CT indicating that the therapeutic response was a stable disease, by day 159, the CTC count had increased to 513 cells $/ 7.5 \mathrm{ml}$. The chemotherapeutic regimen was then changed to cisplatin $\left(23 \mathrm{mg} / \mathrm{m}^{2}\right)$ plus irinotecan $\left(50 \mathrm{mg} / \mathrm{m}^{2}\right)$, but this subsequently failed due to the progression of lumbar spine metastasis, as identified by magnetic resonance imaging. The patient survived for a total of 246 days, but finally succumbed to respiratory failure due to cancerous lymphangitis.

\section{Discussion}

CTCs may not be detected in a number of advanced gastric cancer cases, in contrast to breast and prostate cancers $(15,16)$. When the markers to capture CTC are changed from epithelial cell adhesion molecules to cytokeratins, circulating gastric cancer cells are only detected in $11.6-15.5 \%$ of cases (17). A recent study revealed that gastric CTCs were detected in 28/42 cases using the CellSearch ${ }^{\circledR}$ system (18). In particular, a high number of CTCs (30-18,015 cells/7.5 ml) were detected in 
all 5/5 cases with bone marrow metastases. The bone marrow is considered a reservoir for disseminated tumor cells (19). Thus, gastric cancer subtypes that are characterized by multiple bone metastases and/or DIC may be associated with high numbers of CTC, as observed in the present two cases. The high CTC count exhibited in such patients may be useful to monitor therapeutic effect. Regarding the cases described in the present study, the change in the CTC count was considered to be the earliest marker applicable in current practice. In case 1, an effective chemotherapy regimen was conducted, however, the CA19-9 level was elevated for the first 16 days then decreased. By contrast, the CTC count had decreased to $<1 \%$ of the pre-treatment level by the 17th day. The CTC count served as a beneficial marker, and functioned as a determining factor aiding the decision on whether treatment should be continued or not. Furthermore, once the CTC count had recovered to the pre-treatment level, CA19-9 remained at a low level. Clinical symptoms, including bone pain, worsened gradually and later CT imaging indicated disease progression. The other tumor marker, CEA, was also ineffective in this case. In case 2 , the CTC count had decreased to $<3 \%$ of the pre-treatment level on the 11th day after treatment. Simultaneously, the values of CEA and CA19-9 were measured, however, they demonstrated differential readings. Additionally, on the 159th day, when the CTC count reached $218 \%$ of the initial level, the values of CEA and CA19-9 remained below the initial levels. Such evidence demonstrates that the CTC count may function as a definitive and instant marker without a time lag, as shown previously $(15,16)$.

In conclusion, the CTC count may serve as a reliable predictive biomarker of therapeutic response for gastric cancer subgroups, characterized by multiple bone metastases and/or DIC. CTC count may also present a useful tool for molecular biological analysis. Further confirmatory studies are warranted to investigate the detection rate of CTCs in this particular subgroup of advanced gastric cancer with bone metastasis and/or DIC.

\section{Acknowledgements}

The authors would like to thank Enago (www.enago.jp) for reviewing the English language of the original manuscript.

\section{References}

1. Yoshikawa $\mathrm{K}$ and Kitaoka $\mathrm{H}$ : Bone metastasis of gastric cancer. Jpn J Surg 13: 173-176, 1983.

2. Silvestris N, Pantano F, Ibrahim T, Gamucci $T$, De Vita F, Di Palma T, Pedrazzoli P, Barni S, Bernardo A, Febbraro A, et al: Natural history of malignant bone disease in gastric cancer: Final results of a multicenter bone metastasis survey. PLoS One 8: e74402, 2013.

3. Rhee J, Han SW, Oh DY, Im SA, Kim TY and Bang YJ: Clinicopathologic features and clinical outcomes of gastric cancer that initially presents with disseminated intravascular coagulation: A retrospective study. J Gastroenterol Hepatol 25: $1537-1542,2010$.

4. Takashima A, Shirao K, Hirashima Y, Takahari D, Okita NT, Nakajima TE, Kato K, Hamaguchi T, Yamada Y and Shimada Y: Sequential chemotherapy with methotrexate and 5-fluorouracil for chemotherapy-naive advanced gastric cancer with disseminated intravascular coagulation at initial diagnosis. J Cancer Res Clin Oncol 136: 243-248, 2010
5. Etoh T, Baba H, Taketomi A, Nakashima H, Kohnoe S, Seo Y, Fukuda T and Tomoda H: Diffuse bone metastasis with hematologic disorders from gastric cancer: Clinicopathological features and prognosis. Oncol Rep 6: 601-605, 1999.

6. Etoh T, Baba H, Taketomi A, Nakashima H, Kohnoe S, Seo Y, Saito T and Tomoda H: Sequential methothrextate and 5 -fuororacil therapy for diffuse bone metastasis from gastric cancer. Anticancer Res 18: 2085-2088, 1998.

7. Kang BW, Kim JG, Kwon OK, Chung HY and $\mathrm{Yu}$ W: Non-platinum-based chemotherapy for treatment of advanced gastric cancer: 5-fluorouracil, taxanes, and irinotecan. World J Gastroenterol 20: 5396-5402, 2014.

8. Boku N, Yamamoto S, Fukuda H, Shirao K, Doi T, Sawaki A, Koizumi W, Saito H, Yamaguchi K, Takiuchi H, et al; Gastrointestinal Oncology Study Group of the Japan Clinical Oncology Group: Fluorouracil versus combination of irinotecan plus cisplatin versus S-1 in metastatic gastric cancer: A randomised phase 3 study. Lancet Oncol 10: 10631069, 2009.

9. Cao C, Zhang X, Kuang M, Gu D, He M, Chen J and Tang C: Survival benefit from $\mathrm{S}-1$ as compared to Fluorouracil in Asian patients with advanced gastrointestinal cancer: A metaanalysis. Cancer Sci 105: 1008-1014, 2014

10. Bang YJ, Van Cutsem E, Feyereislova A, Chung HC, Shen L, Sawaki A, Lordick F, Ohtsu A, Omuro Y, Satoh T, et al; ToGA Trial Investigators: Trastuzumab in combination with chemotherapy versus chemotherapy alone for treatment of HER2-positive advanced gastric or gastro-oesophageal junction cancer (ToGA): A phase 3, open-label, randomised controlled trial. Lancet 376: 687-697, 2010.

11. Hiraiwa K, Takeuchi H, Hasegawa H, Saikawa Y, Suda K, Ando T, Kumagai K, Irino T, Yoshikawa T, Matsuda S, et al: Clinical significance of circulating tumor cells in blood from patients with gastrointestinal cancers. Ann Surg Oncol 15: 3092-3100, 2008 .

12. Otsuka K, Imai H, Soeda H, Komine K, Ishioka C and Shibata H: Practical utility of circulating tumour cells as biomarkers in cancer chemotherapy for advanced colorectal cancer. Anticancer Res 33: 625-629, 2013.

13. Komine K, Inoue M, Otsuka K, Fukuda K, Nanjo H and Shibata H: Utility of measuring circulating tumor cell counts to assess the efficacy of treatment for carcinomas of unknown primary origin. Anticancer Res 34: 3165-3168, 2014.

14. Eisenhauer EA, Therasse P, Bogaerts J, Schwartz LH, Sargent D, Ford R, Dancey J, Arbuck S, Gwyther S, Mooney M, et al: New response evaluation criteria in solid tumours: Revised RECIST guideline (version 1.1). Eur J Cancer 45: 228-247, 2009.

15. Cristofanilli M, Budd GT, Ellis MJ, Stopeck A, Matera J, Miller MC, Reuben JM, Doyle GV, Allard WJ, Terstappen LW and Hayes DF: Circulating tumor cells, disease progression, and survival in metastatic breast cancer. N Engl J Med 351: 781-971, 2004.

16. Moreno JG, O'Hara SM, Gross S, Doyle G, Fritsche H, Gomella LG and Terstappen LW: Changes in circulating carcinoma cells in patients with metastatic prostate cancer correlate with disease status. Urology 58: 386-392, 2001.

17. Koga T, Tokunaga E, Sumiyoshi Y, Oki E, Oda S, Takahashi I, Kakeji Y, Baba H and Maehara Y: Detection of circulating gastric cancer cells in peripheral blood using real time quantitative RT-PCR. Hepatogastroenterology 55: 1131-1135, 2008.

18. Toyoshima K, Hayashi A, Kashiwagi M, Hayashi N, Iwatsuki M, Ishimoto T, Baba Y, Baba $\mathrm{H}$ and Ohta $\mathrm{Y}$ : Analysis of circulating tumor cells derived from advanced gastric cancer. Int J Cancer 137: 991-998, 2015.

19. Pantel K and Alix-Panabières C: Bone marrow as a reservoir for disseminated tumor cells: A special source for liquid biopsy in cancer patients. Bonekey Rep 3: 584, 2014. 